\title{
The IBM Pluggable Sequence Relay Calculator
}

\section{Introduction}

The first two machines of this type were built during the war for the Aberdeen Proving Ground, were delivered in December 1944 and were in operation during the last eight months of the war. In November 1945 they were returned to the factory for the provision of increased storage capacity and other improvements. At that time three additional machines of the improved type were built-one for the Naval Proving Ground at Dahlgren and two for the Watson Scientific Computing Laboratory at Columbia University. The latter are available to scientists engaged in basic research. The machines were designed and built in the Engineering Department of the IBM by C. D. Lake, B. M. Durfee, and D. R. Piatt.

The machine reads numbers from punched cards, performs a sequence of calculations on them by means of relay networks, and punches the results in cards. The calculating unit is the fastest relay unit that has been announced (November 1947); six-digit multiplications are performed in 0.15 second, which is about three times as fast as that of any other relay multiplying unit. The basic operations include addition, subtraction, multiplication, division, square root, and column shift. There are 36 storage and computing registers; mathematical tables with monotonic argument may be used. The reading and recording speed is greater than that of any other existing calculator. Cards are read and punched at the rate of 100 per minute, and it is possible to read four cards simultaneously and punch a fifth at this rate.

In comparison with the IBM Sequence Controlled Calculator at Harvard, this machine is limited in internal storage capacity, number of significant figures, and flexibility of sequencing; on the other hand, multiplying speed is about 20 times as great, and the use of plugboard facilities and punched cards permits parallel operation (as distinguished from sequence operation), with further gain in efficiency.

While the speed in performing the arithmetical operations is less than that of electronic machines, the over-all production on general computing is extremely high because of the many other factors involved. These factors include the use of automatic control panels for quick change from one problem to another; efficient card reading, punching, and comparing facilities; convenience of operation; and the advantages of having twin machines in the same room. For a great many scientific problems, the internal storage capacity of all existing machines is quickly exceeded, and the over-all speed is determined by the facilities for reading and recording rather than by the speed of computing; in such cases these calculators, which take full advantage of punched card storage, are unequalled in efficiency. A simple example of this type of problem is the manipulation of large harmonic series described in $\$ 6$.

The scope of the machine may be seen by comparing it with the standard IBM Calculating Punch (type 602). The principal differences include 
(a) higher operating speed (in general it is about 10 times as fast as the Calculating Punch; additions are performed at the rate of 40 per second, and multiplications at the rate of 6 per second),

(b) greater storage capacity (it has 36 storage and computing registers of 6 or 12 digits each, permitting more complicated sequences with one run of the cards),

(c) more flexible card reading and punching facilities (it has two separate card feeding units with four reading stations; it can punch 100 eightycolumn cards per minute),

(d) additional functions (square root is included; one card feed can be used for a mathematical table; the machine performs many functions of the collator and reproducer),

(e) fewer significant figures (basic capacity of the multiplier is $6 \times 6$ $=12 ; 12 \times 12=24$ may be performed by sequencing).

\section{Card Reading and Punching}

The card reading unit is similar to a standard IBM Reproducing Punch, except that a full reading station has been inserted ahead of the punching station to replace the conventional $x$-brushes. Cards in feed A pass a set of $\mathrm{x}$-brushes and two complete reading stations, " 1 " and " 2 "; cards in feed B pass reading station " 3 ," the punching station, and reading station " 4 ."

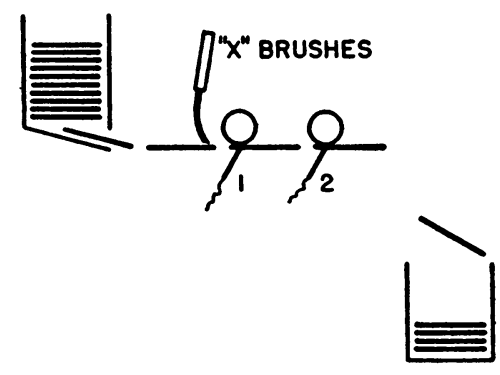

FEED A

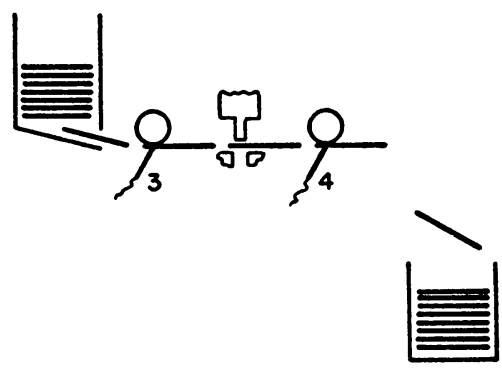

FEED B

Figure 1

Data may be read from as many as 4 cards simultaneously and results punched on a fifth. Data may also be passed back from brush station " 4 " to the following card so that data from earlier cards in the series may also be available. The position of the $x$-brushes is such that they are reading "y" from one card at the same time that brushes " 1 " are reading the 9 of the preceding card. The two feeds may be operated continuously or intermittently, in synchronism or either one independently, as directed by the sequence control. The feed operates at the rate of 100 cards per minute (.6 second per card). A digit selector is included, and 24 dial switches provide for the storage in the machine of constant factors aggregating 24 digits.

There is a comparing unit of 80 columns, of the type used on the reproducing punch, which may be plugged to reading brushes or registers to stop the machine when the factors being compared are unequal. The comparing unit may be used to check the results of a previous run or to stop an itera- 
tive process. It has pluggable outlets which allow it to be split into 8 independent units. Additional comparing and collating facilities will be listed in the next paragraph.

\section{Relay Groups}

The storage and computing units consist entirely of electromechanical relays arranged in 36 groups. These relay groups perform nine principal functions:

(a) receiving data from the cards

(b) receiving data from another relay group or from storage switches

(c) transmitting data to another group

(d) transmitting data to the punch magnets for recording on the cards or to the comparing magnets

(e) operating in conjunction with another group to form the sum or difference of the numbers in the groups

(f) operating in conjunction with other groups to form products

(g) operating in conjunction with other groups to form quotients

(h) operating in conjunction with other groups to form square roots

(i) operating in conjunction with another group to compare two numbers and indicate high-low-equal.

Each group is capable of performing two or more of these functions as shown in table 1 (the switch storage units are also shown). Some of the transfers of data take place between definite groups, and some can be performed at will over one of two main channels or "busses." Provision is made for connecting the two busses momentarily for transfers. The first column of the table lists the relay groups and the switch storage groups; the 12-digit groups are shown in two sections, 1-6 and 7-12. The next four columns indicate which groups can receive data from cards, from bus 1 , from bus 2 , and from other groups by direct connection. The following three columns indicate which groups may transmit data to cards, bus 1 , and bus 2 . In columns 9-12 are shown the groups which are used in the operations of cross addition (CA), multiplication (MP), division (Div), and square root ( $\mathrm{Sq} \mathrm{Rt}$ ). A bracket in the columns headed Bus 1 and Bus 2 means that the result of a cross-adding operation is transmitted to the bus; a bracket in the direct transfer column indicates that two receiving groups (shown in the first column) are involved; a bracket in the CA column indicates the groups which may be cross-added. The final column indicates groups which are capable of transmitting complements or true figures as desired. Groups 19 and 20 and groups 24 and 25 can be used to compare two numbers for highlow-equal.

\section{Basic Operations}

The operations of all parts of the machine are synchronized with a rotating shaft in the card reading unit. This shaft turns at the rate of 100 revolutions per minute. When the card feeds are operated, they are moved in synchronism with this shaft so that a card is read or punched during one revolution of the shaft. During a revolution of the shaft a timing circuit produces 48 impulses (at 0.0125 -second intervals) which may be used to operate relays, and a second timing circuit produces 14 impulses 
which are used for reading and punching cards. Thus, 48 relay impulses are equivalent to 14 card pulses, or one card impulse is equivalent to $3 \frac{3}{7}$ relay impulses. The cards are fed top or 12's edge first, and the 0 card impulse corre-

CARD IMPULSES

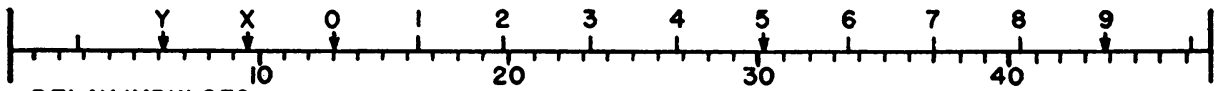

RELAY IMPULSES

Figure 2

sponds to the 13 relay impulse, as shown in figure 2. All relays are picked up and dropped out by the relay pulses, and all card reading, punching, and comparing are controlled by the card pulses.

Table 1

\begin{tabular}{|c|c|c|c|c|c|c|c|c|c|c|c|c|}
\hline \multirow{3}{*}{ Group } & \multicolumn{4}{|c|}{ Receive from } & \multicolumn{3}{|c|}{ Transmit to } & \multirow{3}{*}{$\mathrm{CA}$} & \multirow{3}{*}{ MP } & \multirow{3}{*}{ Div } & \multirow{3}{*}{ Sq Rt } & \multirow{3}{*}{$\begin{array}{l}\text { Inv } \\
\text { Read } \\
\text { Out }\end{array}$} \\
\hline & \multirow{2}{*}{ Cards } & \multicolumn{2}{|c|}{ Bus } & \multirow{2}{*}{$\begin{array}{c}\text { Direct } \\
\text { Transfer }\end{array}$} & \multirow{2}{*}{ Cards } & \multicolumn{2}{|c|}{ Bus } & & & & & \\
\hline & & 1 & 2 & & & 1 & 2 & & & & & \\
\hline MC & $\sqrt{ }$ & & $\sqrt{ }$ & & & & $\sqrt{ }$ & & $\sqrt{ }$ & & & $\sqrt{ }$ \\
\hline MP & $\sqrt{ }$ & $\sqrt{ }$ & & & & $\sqrt{ }$ & & & $\sqrt{ }$ & & & $\sqrt{ }$ \\
\hline 1 & $\sqrt{ }$ & & & & & & & $\sqrt{ }$ & $\sqrt{ }$ & & & \\
\hline 2 & $\sqrt{ }$ & & & & & & & $\sqrt{ })$ & $\sqrt{ }$ & & & \\
\hline 3 & $\sqrt{ }$ & & & & & & & $\sqrt{ }$ & $\sqrt{ }$ & & & \\
\hline 4 & $\sqrt{ }$ & & & & & & & $\sqrt{ })$ & $\sqrt{ }$ & & & \\
\hline 5 & $\sqrt{ }$ & & & & & & & $\sqrt{ }$ & $\sqrt{ }$ & & & \\
\hline 6 & $\sqrt{ }$ & & & & & & & $\sqrt{ })$ & $\sqrt{ }$ & & & \\
\hline 7 & & & & & & & & & $\sqrt{ }$ & & & \\
\hline 8 & & & & & & & & & $\sqrt{ }$ & & & \\
\hline 9 & $\sqrt{ }$ & $\sqrt{ }$ & & $\begin{array}{c}11+12 \\
(7-12)\end{array}$ & & $\sqrt{ }$ & & $\sqrt{ }$ & & $\sqrt{ }$ & & \\
\hline 10 & $\sqrt{ }$ & & $\sqrt{ }$ & 13 & & & $\sqrt{ }$ & $\sqrt{ })$ & & & & \\
\hline $11(1-6)$ & $\sqrt{ }$ & $\sqrt{ }$ & & & & & & $\sqrt{ }$ & & $\sqrt{ }$ & $\sqrt{ }$ & \\
\hline$(7-12)$ & $\sqrt{ }$ & & $\sqrt{ }$ & $9+10$ & & $\left\{\begin{array}{l}\sqrt{ } \\
(1-6)\end{array}\right.$ & & $\sqrt{ }$ & & $\sqrt{ }$ & $\sqrt{ }$ & \\
\hline $12(1-6)$ & $\sqrt{ }$ & $\sqrt{ }$ & & & & & $\sqrt{ }$ & $\sqrt{ }$ & & $\sqrt{ }$ & $\sqrt{ }$ & \\
\hline$(7-12)$ & $\sqrt{ }$ & & $\sqrt{ }$ & & & & & $\sqrt{ })$ & & $\sqrt{ }$ & $\sqrt{ }$ & \\
\hline 13 & $\sqrt{ }$ & & $\sqrt{ }$ & $9+10$ & $\sqrt{ }$ & & $\sqrt{ }$ & & & $\sqrt{ }$ & & $\sqrt{ }$ \\
\hline 14 & & & $\sqrt{ }$ & & & & $\sqrt{ }$ & & & & & $\sqrt{ }$ \\
\hline 15 & & & $\sqrt{ }$ & & & & $\sqrt{ }$ & & & & & $\sqrt{ }$ \\
\hline
\end{tabular}


Table 1 (cont.)

\begin{tabular}{|c|c|c|c|c|c|c|c|c|c|c|c|c|}
\hline \multirow{3}{*}{ Group } & \multicolumn{4}{|c|}{ Receive from } & \multicolumn{3}{|c|}{ Transmit to } & \multirow{3}{*}{$\mathrm{CA}$} & \multirow{3}{*}{ MP } & \multirow{3}{*}{ Div } & \multirow{3}{*}{ Sq Rt } & \multirow{3}{*}{\begin{tabular}{|l} 
Inv \\
Read \\
Out
\end{tabular}} \\
\hline & \multirow{2}{*}{ Cards } & \multicolumn{2}{|c|}{ Bus } & \multirow{2}{*}{$\begin{array}{l}\text { Direct } \\
\text { Transfer }\end{array}$} & \multirow{2}{*}{ Cards } & \multicolumn{2}{|c|}{ Bus } & & & & & \\
\hline & & 1 & 2 & & & 1 & 2 & & & & & \\
\hline $16(1-6)$ & $\sqrt{ }$ & $\sqrt{ }$ & & $11+11$ & $\sqrt{ }$ & $\sqrt{ }$ & & & $\sqrt{ }$ & $\sqrt{ }$ & $\sqrt{ }$ & $\sqrt{ }$ \\
\hline$(7-12)$ & $\sqrt{ }$ & & $\sqrt{ }$ & 11T12 & $\sqrt{ }$ & & $\sqrt{ }$ & & $\sqrt{ }$ & $\sqrt{ }$ & $\sqrt{ }$ & $\sqrt{ }$ \\
\hline $17(1-6)$ & & $\sqrt{ }$ & & & $\sqrt{ }$ & $\sqrt{ }$ & & & & $\sqrt{ }$ & & $\sqrt{ }$ \\
\hline$(7-12)$ & & & $\sqrt{ }$ & & $\sqrt{ }$ & & $\sqrt{ }$ & & & $\sqrt{ }$ & & $\sqrt{ }$ \\
\hline $18(1-6)$ & & $\sqrt{ }$ & & & $\sqrt{ }$ & $\sqrt{ }$ & & & & $\sqrt{ }$ & & $\sqrt{ }$ \\
\hline$(7-12)$ & & & $\sqrt{ }$ & & $\sqrt{ }$ & & $\sqrt{ }$ & & & $\sqrt{ }$ & & $\sqrt{ }$ \\
\hline 19 & $\sqrt{ }$ & $\sqrt{ }$ & & & & $\sqrt{ }$ & & & & & & $\sqrt{ }$ \\
\hline 20 & $\sqrt{ }$ & $\sqrt{ }$ & & & & $\sqrt{ }$ & & & & & & $\sqrt{ }$ \\
\hline 21 & $\sqrt{ }$ & $\sqrt{ }$ & & & $\sqrt{ }$ & $\sqrt{ }$ & & & & & & $\sqrt{ }$ \\
\hline 22 & & $\sqrt{ }$ & & & $\sqrt{ }$ & $\sqrt{ }$ & & & & & & $\sqrt{ }$ \\
\hline 23 & & $\sqrt{ }$ & & & $\sqrt{ }$ & $\sqrt{ }$ & & & & & & $\sqrt{ }$ \\
\hline 24 & $\sqrt{ }$ & & $\sqrt{ }$ & & & & $\sqrt{ }$ & & & & & $\sqrt{ }$ \\
\hline 25 & $\sqrt{ }$ & & $\sqrt{ }$ & & & & $\sqrt{ }$ & & & & & $\sqrt{ }$ \\
\hline 26 & $\sqrt{ }$ & & $\sqrt{ }$ & & $\sqrt{ }$ & & $\sqrt{ }$ & & & & & $\sqrt{ }$ \\
\hline 27 & & & $\sqrt{ }$ & & $\sqrt{ }$ & & $\sqrt{ }$ & & & & & $\sqrt{ }$ \\
\hline 28 & & & $\sqrt{ }$ & & $\sqrt{ }$ & & $\sqrt{ }$ & & & & & $\sqrt{ }$ \\
\hline Sq Rt & & & & & & $\sqrt{ }$ & & & & & $\sqrt{ }$ & \\
\hline Sw 1 & & & & & & $\sqrt{ }$ & & & & & & $\sqrt{ }$ \\
\hline Sw 2 & & & & & & $\sqrt{ }$ & & & & & & $\sqrt{ }$ \\
\hline Sw 3 & & & & & & & $\sqrt{ }$ & & & & & $\sqrt{ }$ \\
\hline Sw 4 & & & & & & & $\sqrt{ }$ & & & & & $\sqrt{ }$ \\
\hline
\end{tabular}

The various relay groups performing the different functions can be plugged to operate on selected relay pulses as shown in figure 3 . These operations include in and out for transfers; resetting; and starting to cross-add, multiply, divide, extract the square root, and interrupt a sequence (see §5).

The relays operate on a pentad system with a relay representing each digit from 1 to 5 . In transferring a number from one group of relays to another, a separate conductor is used for each relay to permit simultaneous operation; five conductors are therefore required for one decimal digit, or 30 for a six-digit number. This mode of operation gives high speed (a single impulse of 0.0125 second is sufficient to set up a given relay configuration or to reset a group of relays) but increases the number of conductors and switches required. 


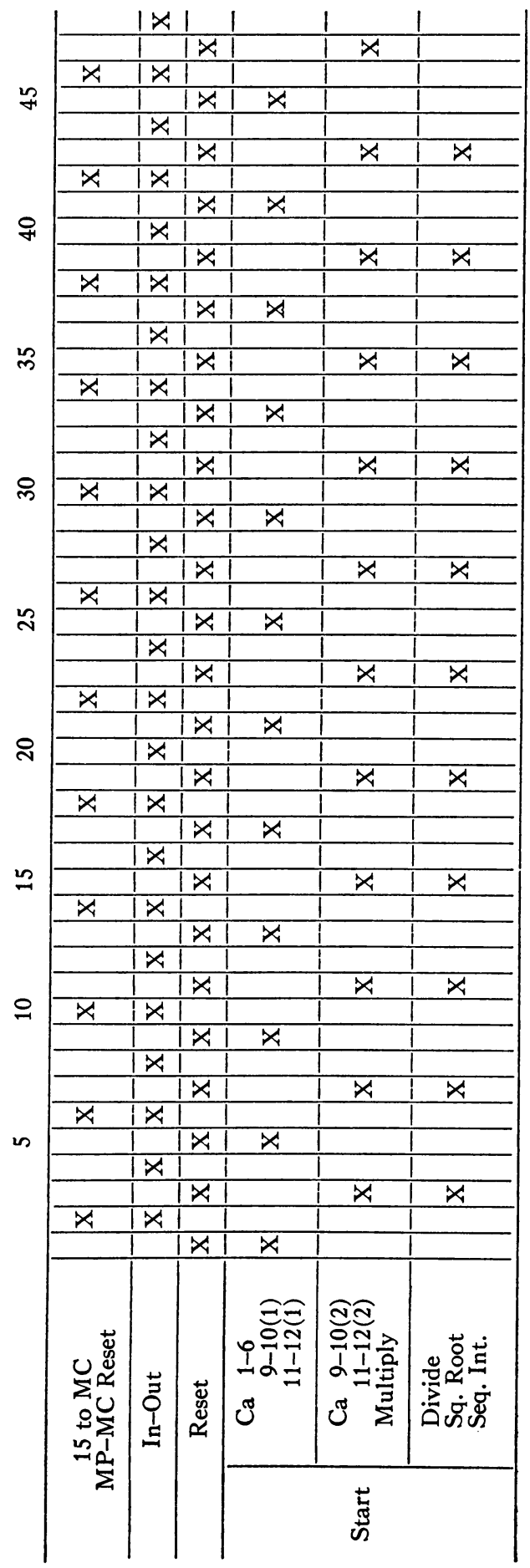


The actual operations of addition or subtraction require two impulses, but four impulses are required between successive additions or subtractions involving the same relay group. Addition or subtraction involves 3 relay groups, say A, B, and C, and the operation is as follows:

Impulse 1, 2: read quantities into $\mathrm{A}$ and $\mathrm{B}$

3 : test for carries

3, 4 : read sum or difference into $C$

4 : reset $A$ and $B$

5, 6: read new quantities into $A$ and $B$; transfer data in $C$ elsewhere 6: clear C.

The arrangements of the relay groups for cross-adding are shown in table 1 ; the operation can take place simultaneously in several independent triads of groups. By using four groups (a, b, c, d), a series of numbers can be added at the rate of 40 per second; the operations are:

$$
\text { (a) }+(\mathrm{b}) \rightarrow \mathrm{c}, \quad \mathrm{c}+(\mathrm{d}) \rightarrow \mathrm{b}, \quad(\mathrm{a})+\mathrm{b} \rightarrow \mathrm{c}, \text { etc. },
$$

where new quantities read into the groups are indicated by parentheses.

Multiplication is performed by addition of left- and right-hand components in a manner analogous to that in the IBM Multiplying Punch (type 601); the details are different largely because of the relay method of addition. The right and left components are combined for each digit of the multiplier, and column shift is performed after the components are combined. Alternate sets of relay groups are employed for alternate digits of the multiplier. As shown in table 1, relay groups multiplier, multiplicand, numbers $1-8$, and 16 are involved in the process which proceeds as follows (see table 2):

1. Read factors (MP and MC) into multiplier and multiplicand registers.

2. Form right- and left-hand components of multiplicand and first digit of multiplier (RC1 and LC1).

3. Add RC1 and LC1 to give the complete partial product of the multiplicand by the first digit of the multiplier, (p1). (Similarly form other partial products, $\mathrm{p} 2$ to $\mathrm{p} 6$.)

4. Combine the various partial products employing addition and column shift.

The operations of the registers are as shown in table 2 (note that each operation involves two impulses).

As shown in the table, successive multiplications may be spaced at intervals of $12 \times 0^{\mathrm{s}} .0125=0^{\mathrm{s}} .15$, provided full advantage is taken of overlap. A single multiplication without overlap requires .2 second.

Division is performed by subtracting the divisor, or five times the divisor, repeatedly from the dividend. The dividend is shifted to the left after the completion of the operation in each position. Subtraction is performed by adding the complement of the number to be subtracted. In adding the complement a carry will occur through the highest order, provided the number being subtracted is less than the one from which it is subtracted. This carry is sensed in advance so that the addition takes place only when desired; in other cases the shift of the dividend takes place instead. The 
average dividing time is .2 second per quotient digit. There is capacity for 12 figures in the dividend, 6 in the divisor, and 10 in the quotient.

Square root is extracted by successive additions of odd numbers to the complement of the original number. There is capacity for 12 figures in the original number and 6 in the square root.

Table 2

\begin{tabular}{|c|c|c|c|c|c|c|c|c|c|c|c|}
\hline \multirow{2}{*}{ Impulses } & \multicolumn{11}{|c|}{ Registers } \\
\hline & MP & MC & 1 & 2 & 3 & 4 & 5 & 6 & 7 & 8 & 16 \\
\hline $\begin{array}{l}1 \\
2\end{array}$ & MP & $\mathrm{MC}$ & $\mathrm{RC} 1$ & LC1 & & & & & & & \\
\hline $\begin{array}{l}3 \\
4\end{array}$ & & & & & $\mathrm{RC} 2$ & LC2 & & $\mathrm{p} 1$ & & & \\
\hline $\begin{array}{l}5 \\
6\end{array}$ & & & RC3 & LC3 & & & & & p2 & P1 & \\
\hline $\begin{array}{l}7 \\
8\end{array}$ & & & & & $\mathrm{RC4}$ & LC4 & P2 & p3 & & & \\
\hline $\begin{array}{r}9 \\
10\end{array}$ & & & $\mathrm{RC5}$ & LC5 & & & & & $\mathrm{p} 4$ & P3 & \\
\hline $\begin{array}{l}11 \\
12\end{array}$ & & & & & RC6 & LC6 & P4 & $\mathrm{p} 5$ & & & \\
\hline $\begin{array}{l}13 \\
14\end{array}$ & MP & MC & $\mathrm{RC} 1$ & LC1 & & & & & p6 & P5 & \\
\hline $\begin{array}{l}15 \\
16\end{array}$ & & & & & $\mathrm{RC} 2$ & LC2 & $\mathrm{p} 1$ & & & & P6 \\
\hline
\end{tabular}

$\mathrm{Pi}=\mathrm{p} 1+\mathrm{p} 2+\cdots+\mathrm{pi}$

Numbers read from relay groups 13 and 16 may be shifted to the right or left. They are provided with pluggable output which permits any arbitrary shift; group 16 has two such sets of outlets. An automatic shift of 1,2 , or 3 columns to the right or left from group 16 may also be accomplished by plugging a single control wire.

Rounding of positive numbers may be accomplished in any adding group by adding 5 . A credit balance may be rounded by wiring registers 11,12 , and 16 to add or subtract 5 according to the sign of the balance.

Card feed $A$ and the relay comparing unit can be used for consulting mathematical tables. The table cards are fed at the rate of 100 cards per minute, so that long searches should be avoided by having the table cards in the same order or by using a short table with fairly long interpolation procedure. The first method is illustrated in $\$ 6$. By combining field selection with card selection in a carefully constructed table, it is possible to consult a fairly extensive table in a few seconds.

\section{Sequence Control}

The basic operations of the machine are initiated by a pluggable sequence and to some extent by the punching on the cards. The latter include the 
usual functions of identification of algebraic sign, the use of a digit emitter, rate card operation, and group control.

The pluggable sequence on the control panels initiates most of the operations. There are 96 sequencing relays with outlets to the plugboard so that impulses through them can be used for control purposes. These relays operate in succession, actuated by the successive relay impulses from the main timing circuit mentioned in a previous paragraph. The complete sequence of 96 relays is operated in two revolutions of the shaft, or a single cycle involving only 48 relays may be employed. When operating on the singlecycle basis, a card is fed during each revolution; on the double-cycle basis a card is fed on the first revolution of each successive pair of revolutions. The sequence can be temporarily suspended and resumed at the same point one revolution (48 impulses) later.

There are 25 selectors having a total of 132 circuits, with pluggable primary and secondary circuits. These selectors are used for combining and analyzing control impulses, and for routing digits under such control.

Operations such as multiplication, where the necessary number of cycles can be predicted in advance, can be plugged to give maximum efficiency of operation. In operations such as division, square root, table look-up, etc., where the required time cannot be predicted, the sequence is interrupted at the start of the operation and resumed when completed. By combining selectors with the sequencing relays it is possible to alter the sequence on successive revolutions so as to obtain a long sequence, limited only by the capacity of the relays available.

There are two control panels on the machine: one, of three sections, which in general determines the channels through which the data flow, and the other, of two sections, which determines the sequence of operations.

As shown in figure 2, numbers are read from, and punched on, the card during relay impulses 13 to 44 ; consequently, numbers read from a given card during impulses 13 to 44 may be used for computations during impulses 45 to 48 of that cycle and 1 to 12 of the next, and the result may be punched on the same card while the following card is being read. Since a multiplication requires 16 impulses, there is just time to perform a single multiplication on a card and record the answer on the same card (100 cards per minute). A number of cross-additions may be performed simultaneously, but there is no time to cross-add to the product. The sign of the product is determined in time for $x$ or $y$ punching.

Where the results need not be punched on the card from which the factors were read, it is possible to store them in the machine for the next card and thus use the entire card cycle for computation. In this manner it is possible to form 4 products and to do cross-adding at the rate of 100 cards per minute, with some limitations growing out of the available storage.

In comparatively long sequences, cards may be fed on every second cycle, third cycle, etc., the limit to the length of the sequence being determined by the internal storage capacity.

As an example of the control of the operations of the IBM Pluggable Sequence Relay Calculator, consider the problem of forming 4 products and their sum. The 8 factors are on one card in feed $A$, and the results are punched on another card in feed $\mathrm{B}$. The operations require 2 cycles, or 96 impulses, and can be performed at the rate of 50 cards per minute. 
Sixteen impulses after the multiplication is started, the product appears in register 16. It is possible to start a new multiplication every twelfth impulse if the multiplicand is transferred to register 15 and the " 15 to $\mathrm{MC}$ " transfer is used.

The registers for the factors are selected to permit reading of all multipliers on bus 1 and all multiplicands on bus 2 . The products are transferred from register 16 to a 12-digit register or to two 6-digit registers, where they are stored for punching during impulses (6-44) of the following cycle.

\section{Table 3}

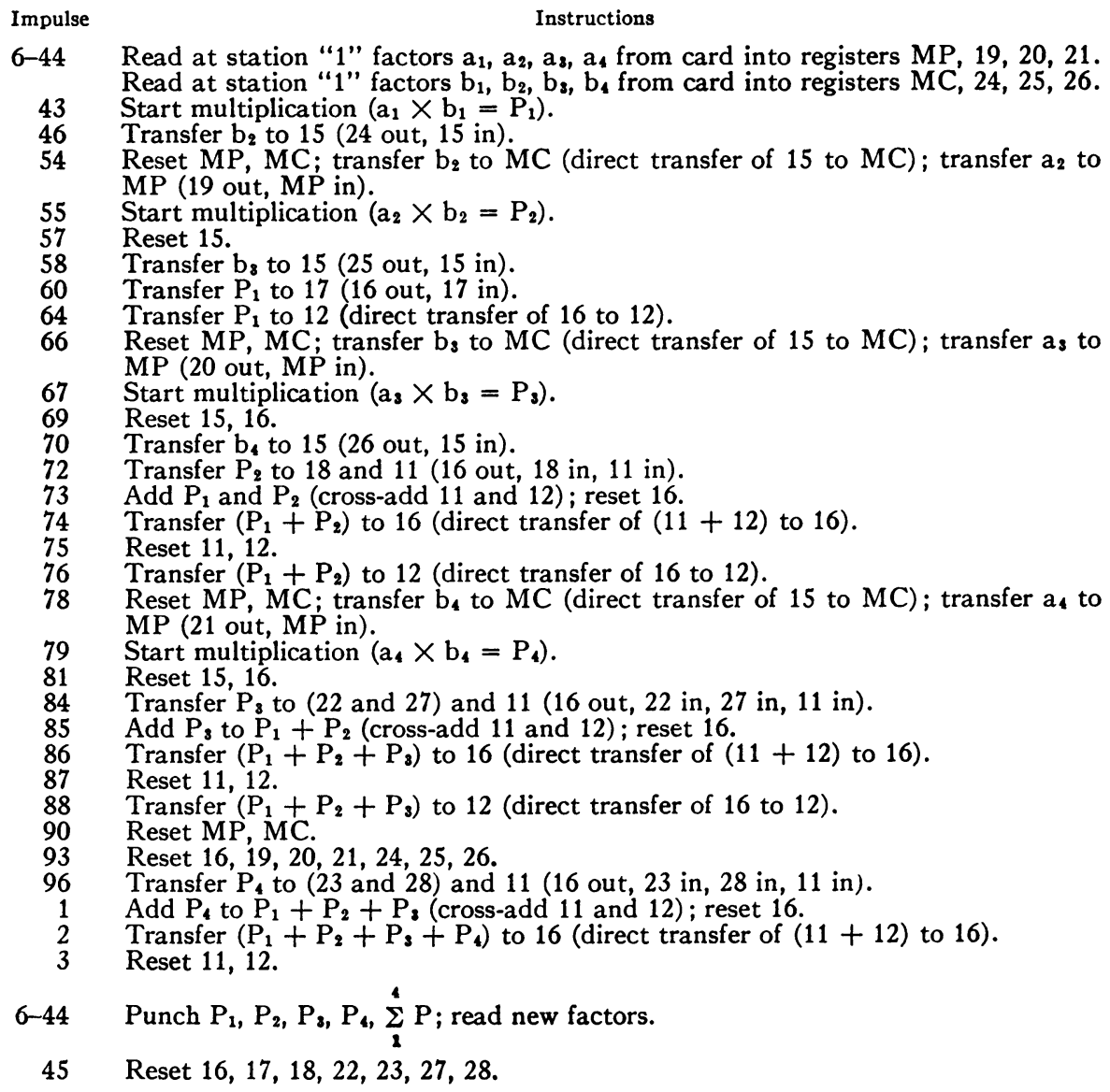

Since a 12-digit sum is assumed, registers 11 and 12 are used to cross-add the products, and each sum is transferred into register 16 because there is a direct connection. After registers 11 and 12 are reset, the accumulated products are transferred back to register 12 for the next cross-addition. The final summation appears in register 16 just before punching time. Note that while the cards are being punched, the new entries are made for the following card (see figure 1). Table 3 indicates the necessary wiring. 


\section{Applications}

The operation of the machines will be illustrated by a few typical examples: the reduction of observational data, the multiplication of matrices and of harmonic series, the solution of differential equations, and the construction of mathematical tables.

In many fields of science and engineering great masses of data are recorded and must be subjected to mathematical treatment in preparation for proper examination and discussion. The efficiency of the punched card for this work is well known and has been utilized in extensive programs at many computing laboratories. ${ }^{1}$ Because of the ability of the relay calculators to eliminate collating, reproducing, and recording operations, they bring greatly increased efficiency to this field. In a recent program a series of 50,000 measurements, $x$ and $y$, were corrected by the following formulae

$$
\begin{aligned}
& \alpha=k+c+b y+x\left(a-0.016+g y+p x+0.002 x^{2}+0.002 y^{2}\right) \\
& \delta=k^{\prime}+c^{\prime}+a^{\prime} x+y\left(b^{\prime}-0.016+p^{\prime} x+g^{\prime} y+0.002 y^{2}+0.002 x^{2}\right)
\end{aligned}
$$

where

$$
\begin{gathered}
k, c, b, a, g, p \\
k^{\prime}, c^{\prime}, b^{\prime}, a^{\prime}, g^{\prime}, p^{\prime}
\end{gathered}
$$

are constant for several hundred values of $x$ and $y$. The cards containing $x$ and $y$ were already completely punched; therefore a new set of cards was prepared during the computation. The values of the constants $k$, $c$, etc. were punched on master cards. The computation was performed in two runs by interchanging $x$ and $y$ on the control panel. The operation proceeds at the rate of 2,000 cards per hour.

A common type of reduction used in science is the following

$$
E=A a+B b+C c+D d
$$

where $A, B, C, D$ are functions of one variable, $x$, and $a, b, c, d$ are functions of another variable, $y$. In this case the cards containing the values of $a, b, c$, $d, x$ would be sorted on $x$ and placed in the punch feed; cards $\mathrm{A}, \mathrm{B}, \mathrm{C}, \mathrm{D}$ would be placed in the read feed (sorted also on $x$ ). The machine would then treat the A, B, C, D cards as a mathematical table and perform the calculation as required.

The multiplication of matrices and of harmonic series are natural applications for these machines. The punched card gives unlimited storage capacity for large matrices or series, and the efficient reading, recording, and highspeed multiplication provide for high-speed operation.

Alt has described ${ }^{2}$ a process whereby the cards containing the elements of the A matrix are placed in one feed, those of the $B$ matrix in the other, and those of the product matrix $C=A B$ are punched on the $B$ cards. The process is continuous and proceeds at the rate of 6,000 cards per hour for $6 \times 6$ multiplication and 3,000 per hour for $12 \times 12$.

By inserting new cards for the $\mathrm{C}$ matrix it would be possible to perform 3 multiplications per card at the rate of 6,000 cards per hour as described in \$4. This would permit 12-digit multiplication (small error in twelfth digit) or the multiplication of 3 separate pairs of matrices on the same cards.

These machines are the most efficient ones in operation for the multiplication of large harmonic series. Consider the case of such a series with 
about 1,000 terms each, coefficients of 12 digits, and 3 or 4 fundamental frequencies. ${ }^{3}$ In forming the product series there are a million possible terms, of which several thousand may have coefficients greater than the rejection limit and therefore will have to be retained. For each of these terms a product (of coefficients) and a sum (of arguments) must be formed. The relay calculator calculates such product terms automatically at the rate of 6,000 sensible terms per hour.

After the individual terms are formed, they are sorted according to the argument and combined to give the resultant series. This operation, which could be performed on the accounting machine and summary punch, is performed on the relay calculator. A linear combination of 2 or more series with some manipulation of the arguments requires a third machine set-up.

The relay calculators perform automatically the solution of ordinary linear differential equations up to and including the sixth order. ${ }^{4}$ In the method used by HaUsman \& Schwarzschild the elements of a sixth-order square matrix are computed, before the integration, for each value of the independent variable. The values of the 6 dependent variables for a given step constitute a matrix with one row of 6 elements. The integration procedure at each step consists of multiplying the square matrix by the singlerow matrix to obtain a new single-row matrix whose elements are the values of the dependent variables for the next value of the independent variable.

In the first integration the fourth differences are neglected. After the first integration the resultant values are used to obtain corrections for these effects by a second run of the cards. On the second integration these corrections are included in the integration. The procedure is thus one of successive approximations of a rapidly converging process.

In applying the method to a set of the fifth order involving about 80 steps, it was found that each integration required about 12 minutes, and about one hour was required for the approximations to converge. The problem involved similar solutions for about 12 sets of initial conditions.

Although the machines have not been used for this purpose, they could be used for the solution of partial differential equations where each new value depends upon a number of values of the old ones. The standard punchedcard machines have been used for such problems, and the relay calculators would provide greater speed and much less handling of the cards.

In the construction of mathematical tables the basic computations for a large number of values of the arguments are usually made at one time. These calculations are regularly performed on the standard machines; additional speed and freedom from card handling make the relay calculators efficient for such problems.

In a set of gear tables recently computed the following formulae were evaluated with one run of the cards, at the rate of about 1,000 values per hour.

$$
\begin{aligned}
& x=A B /(C+D), \quad z=x E F, \\
& y=A B /(C+10 D), \quad w=10 y E F,
\end{aligned}
$$

where $A, B, C, D, E, F$ are given numbers for each entry.

In another set of tables one step involved the solution of the transcendental equation

$$
M=E-e \sin E
$$


where $M$ and $e$ are given and $E$ is required. A small punched-card table gave a rough approximation to $E, E_{0}$. This value $E_{0}$ was used to enter a standard punched-card table of $\sin E$ with tabular interval of $0^{\circ} .01$. From this table were taken $\sin E_{0}$ and $\sin \left(E_{0}+0^{\circ} .01\right)$ by passing the cards through the reproducer in both directions. The relay calculator, with a single run of the cards, evaluated the following formulae:

$$
\begin{aligned}
& \delta_{1}=M-E_{0}+57^{\circ} .2958 e \sin E_{0} \\
& \delta_{2}=M-E_{0}-0^{\circ} .01+57^{\circ} .2958 e \sin \left(E_{0}+0^{\circ} .01\right) \\
& E=E_{0}-0^{\circ} .01 \delta_{1} /\left(\delta_{2}-\delta_{1}\right) .
\end{aligned}
$$

Several members of the Laboratory Staff have assisted in the preparation of this article, especially LiLlian F. HAUSMan, Rebecca Jones, Marjorie Herrick, Phyllis Arnold, and Richard Bennetr. Valuable suggestions were made by GeOfFrey Keller of the Department of Astronomy of Columbia University.

$$
\text { W. J. E. }
$$

1W. J. Eckert, Punched Card Methods in Scientific Computation, The Thomas J. Watson Astronomical Computing Bureau, 1940, chap. $x$.

2 Franz L. Alt, "Multiplication of matrices," $M T A C$, v. 2, p. 12-13.

3 W. J. ECKERT, op. cit., chap. xi.

'L. F. HAusman \& M. SChwarzschild, "Áutomatic integration of linear sixth-order differential equations by means of punched card machines," Rev. Sci. Inst., v. 18, 1947, p. $877,-883$.

\section{Inversion of a Matrix of Order 38}

The general solution of a system of 38 simultaneous linear equations has recently been obtained by the writer, utilizing the Aiken Relay Calculator, constructed for the Naval Proving Ground, Dahlgren, Virginia, by the staff of the Computation Laboratory of Harvard University. The elements of the reciprocal matrix were computed using an adaptation of the GAUsS method of elimination. This method is particularly suited to machine solution in that it permits the use of a few short computing routines, the number of repetitions of which are governed by simple functions of the system order. The fact that a single system of control tapes may be employed to invert a matrix of any order was considered of paramount importance in the choice of this method.

The remarkable feature of the present computation was the degree of accuracy obtained in the elements of the reciprocal matrix. When tested on known data, the inverse matrix yielded results correct to nine significant digits, notwithstanding the fact that the calculator employed is limited to ten significant digits.

The problem solved originated in the field of mathematical economics. It was posed by Professor Wassily W. Leontief ${ }^{1}$ of the Department of Economics in Harvard University. The original system comprises the output-input relations among the industries of the United States, divided into 38 groups, as compiled by the United States Bureau of Labor Statistics for the year 1939. Thirty-eight equations arise, of the form,

$$
A_{i}-\sum_{j=1}^{38} B_{i j}=C_{i}, \quad j \neq i, i=1(1) 38,
$$

